\title{
Isoproterenol-Induced Hypertrophied Rat Hearts: Does Short-Term Treatment Correspond to Long-Term Treatment?
}

\author{
Daisuke TAKESHITA, Juichiro SHIMIZU, Yutaka KITAGAWA, Daisuke YAMASHITA, \\ Kiyoe TOHNE, Chikako NAKAJIMA-TAKENAKA, Haruo ITO, and Miyako TAKAKI \\ Department of Physiology II, Nara Medical University School of Medicine, Nara, 634-8521 Japan
}

\begin{abstract}
In consideration of clinical implications, it is often complained that short-term experimental diseased heart models do not mimic long-term diseased hearts that are often clinically encountered. The aim of the present study was (i) to compare the left ventricular function between rat cardiac hypertrophy models treated with isoproterenol for 3 days (Iso $3 d$ ) and 7 days (Iso 7d) by pressure-volume measurements with a catheter method, and (ii) to follow up the left ventricular function in the same model treated with Iso up to 16 weeks with a less-invasive echocardiography. An infusion of either Iso $\left(1.2 \mathrm{mg} \mathrm{kg}^{-1} \cdot \mathrm{day}^{-1}\right.$ for 3 days-16 weeks) or vehicle (saline $24 \mu$ day $^{-1}$ for 3 days16 weeks; Sa group) was performed by subcutaneously implanting an osmotic minipump. There were no significant differences
\end{abstract}

in the systolic pressure-volume area at midrange left ventricular volume ( $P V A_{m L V v}$ : a mechanical work capability index) between Iso $3 \mathrm{~d}$ and $7 \mathrm{~d}$ groups, though $\mathrm{PVA}_{\mathrm{mLVV}}$ in both groups was significantly reduced from that in the Sa group. From echocardiography, the left ventricular function of the hypertrophy models at 3 days, 1 week, and 2 weeks was unchanged, but the model at a term longer than 4 weeks resulted in prolonged systolic failure. The results indicated that (i) no marked differences in the left ventricular mechanical work capability were found between the Iso $3 \mathrm{~d}$ and $7 \mathrm{~d}$ groups, and that (ii) only a 3-day Iso infusion induced the hypertrophy model similar in shape and function to that induced by a 2-week Iso infusion. We concluded that the 3day model was sufficient.

Key words: conductance catheter, echocardiography, left ventricular volumetry, systolic pressure-volume area.

It is known that chronic infusions of a $\alpha, \beta$-stimulant, norepinephrine, and a $\beta$-stimulant, isoproterenol (Iso), induce cardiac hypertrophy accompanied with enhanced fibrosis among cardiac interstitial cells [1-3]. Many studies on mechanisms underlying Iso-induced cardiac hypertrophy have been reported [4-13]. Cardiac oxidative stress contributes to this type of cardiac remodeling, especially in respect to wall stiffness, based on fibrogenesis in chronically Iso-infused rats $\left(3 \mathrm{mg} \cdot \mathrm{kg}^{-1} \cdot \mathrm{day}^{-1}\right.$ for 10 days), whereas cardiac phosphorylated MAP (mitogen-activated protein) kinases (extracellular signal related kinase; ERK1/2, c-JUN NH$H_{2}$-terminal kinase; JNK, p38) returned to normal [4]. The AT1 (type 1 angiotensin II) receptor plays a crucial role in the development of cardiac hypertrophy and oxidative stress in chronically Iso-infused mice (15 $\mathrm{mg} \cdot \mathrm{kg}^{-1} \cdot \mathrm{day}^{-1}$ for 11 days) [5]. Furthermore, we have recently found that a novel histone deacetylase (HDAC) inhibitor has no effects of antihypertrophic modalities on the Iso-induced rat heart $\left(1.2 \mathrm{mg} \cdot \mathrm{kg}^{-1} \cdot \mathrm{day}^{-1}\right.$ for 3 days) [6]. Nevertheless, the conclusive mechanisms underlying Iso-induced cardiac hypertrophy have not yet been determined [4-13].

We have previously succeeded in inducing cardiac hypertrophy by stimulating the $\beta_{1}$-receptor for only 3 days and have validated the accuracy of left ventricular volumetry in the hypertrophied heart with a conductance catheter system [14]. In consideration of clinical implications, it is often complained that short-term experimental diseased heart models do not mimic long-term diseased hearts that we often clinically encounter. The aim of the

Received on Mar 19, 2008; accepted on May 7, 2008; released online on May 9, 2008; doi:10.2170/physiolsci.RP004508 Abbreviations: $\beta_{1}$ ARs, $\beta_{1}$-adrenergic receptors; Ea, effective arterial resistance: the ratio of end-systolic pressure at end-systolic volume to stroke volume of the left ventricle under stable hemodynamics; $\mathrm{ESP}_{\mathrm{ESV}}$, end-systolic pressure at end-systolic volume; $\mathrm{ESP}_{\mathrm{mLVV}}$, end-systolic pressure at midrange left ventricular volume; Iso $3 \mathrm{~d}$ and Iso $7 \mathrm{~d}$, isoproterenol $1.2 \mathrm{mg} \cdot \mathrm{kg}^{-1} \cdot \mathrm{day}^{-1}$ infusion for 3 days and 7 days; IVSTs and IVSTd, interventricular septum thickness during systole and diastole; LVIDs and LVIDd, left ventricular internal dimensions during systole and diastole; LVPWTs and LVPWTd, left ventricular posterior wall thickness during systole and diastole; NCX1, $\mathrm{Na}^{+} / \mathrm{Ca}^{2+}$ exchanger; PKA, cAMP-dependent protein kinase; PLB, phospholamban; $\mathrm{p}-\mathrm{PLB}$, phospho-Ser ${ }^{16} \mathrm{PLB}$; $\mathrm{PVA}_{\mathrm{mLVV}}$, systolic pressure-volume area at midrange left ventricular volume; RyR2, ryanodine receptor; Sa, Saline infusion $24 \mu \mathrm{l}^{-} \cdot \mathrm{day}^{-1}$ for 3 days-16 weeks; SERCA2, sarcoplasmic reticulum $\mathrm{Ca}^{2+} A T P a s e ; V_{0}$, systolic unstressed volume.

Correspondence should be addressed to Miyako Takaki, Department of Physiology II, Nara Medical University School of Medicine, 840 Shijo-cho, Kashihara, Nara, 634-8521 Japan. Tel: +81-744-29-8829, Fax: +81-744-23-4696, E-mail: mtakaki@naramed-u.ac.jp 


\section{TAKESHITA et al.}

present study was to compare specifically left ventricular function in rat hearts between 3- and 7-day hypertrophy models and to follow up on these models from the same 3day treatment to the long-term 16-week treatment by infusing either Iso $1.2 \mathrm{mg} \cdot \mathrm{kg}^{-1} \cdot \mathrm{day}^{-1}$ (Iso group) or vehicle (saline $24 \mu \mathrm{l} \cdot \mathrm{day}^{-1}$, Sa group), though by different methods.

Previous studies have revealed that the left ventricular end-systolic pressure-volume relation of isolated crosscirculated and in situ ejecting adult rat hearts have upward convex curves, even within the physiological range [1517]. This convex curvature of end-systolic pressure-volume relation has also been described in mice hearts [18, 19]. The systolic pressure-volume area (PVA) obtained from the curved end-systolic pressure-volume relation corresponds to a total mechanical energy per beat and linearly correlates with myocardial oxygen consumption per beat in excised rat hearts [20-22]. Especially, we have proposed PVA at midrange left ventricular volume $\left(\mathrm{PVA}_{\mathrm{mLVV}}\right)$ as a good mechanical work capability index to evaluate the left ventricular function in in situ normal rat hearts [15-17]. Using this index, we found no differences in the reduction of mechanical work capability between 3- and 7-day hypertrophy models. Following up with echocardiography, we also found that the only 3-day hypertrophy model was similar in shape and function to that induced by 2 -week Iso infusion. The results presented here suggested that the 3-day short-term hypertrophy model sufficiently represents the effects of 2-week Iso infusion.

\section{METHODS}

Animals and drug infusion. Experimental procedures followed the regulations of and were approved by the animal care and use committee of Nara Medical University. Male Wistar rats $(n=21)$ weighing 303 to $366 \mathrm{~g}$ ( 8 to 10 weeks) were used in the present experiments. The delivery of drugs was achieved by implanting an osmotic minipump (model 1003D, Alzet, Durect Corp, Cupertino, CA) subcutaneously in the neck under pentobarbital (50 $\mathrm{mg} / \mathrm{kg}$ i.p. $)$ anesthesia. Either Iso $\left(1.2 \mathrm{mg} \cdot \mathrm{kg}^{-1} \cdot \mathrm{day}^{-1}\right.$ for 3 days and 7 days: Iso $3 \mathrm{~d}$ and Iso $7 \mathrm{~d}, n=7$ each), or a vehicle $(0.1 \%$ ascorbic acid in saline $2.4 \mu \mathrm{l} /$ day for 3 days: Sa, $n=7)$ was infused subcutaneously [1]. Osmotic minipumps were removed from the neck 3 or 7 days after
A

Short-term

(I) Sa group $(n=7)$

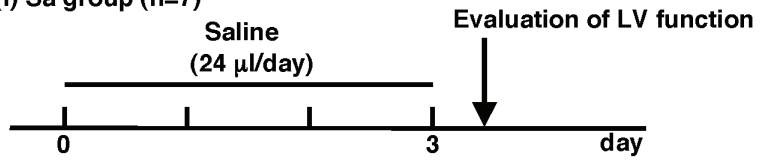

(II) Iso3d, Iso3d(-) groups ( $\mathrm{n}=7$ each)

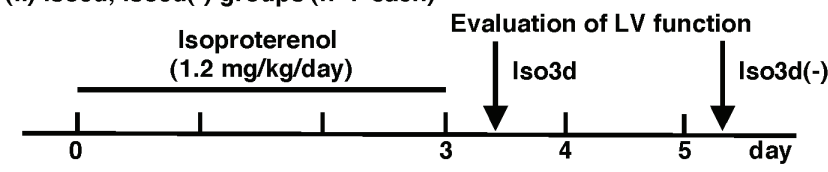

(III) Iso7d, Iso7d(-) groups ( $\mathrm{n}=7$ each)

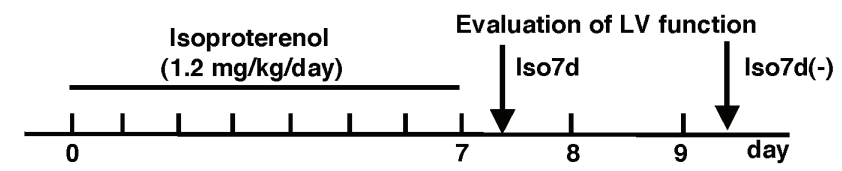

Long-term

(I) Sa group $(n=3) \quad$ Saline

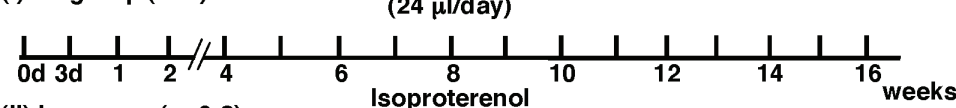

$\begin{array}{ll}\text { (II) Iso group ( } \mathrm{n}=3-8) & (1.2 \mathrm{mg} / \mathrm{kg} / \mathrm{day})\end{array}$

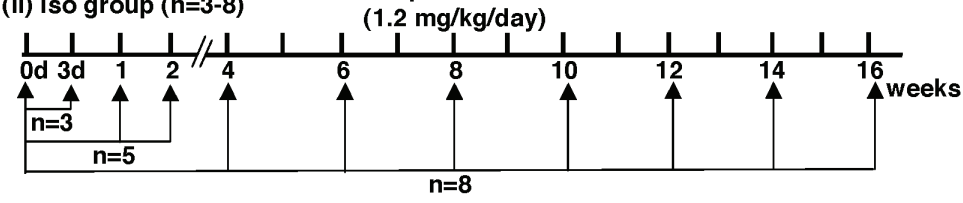

B

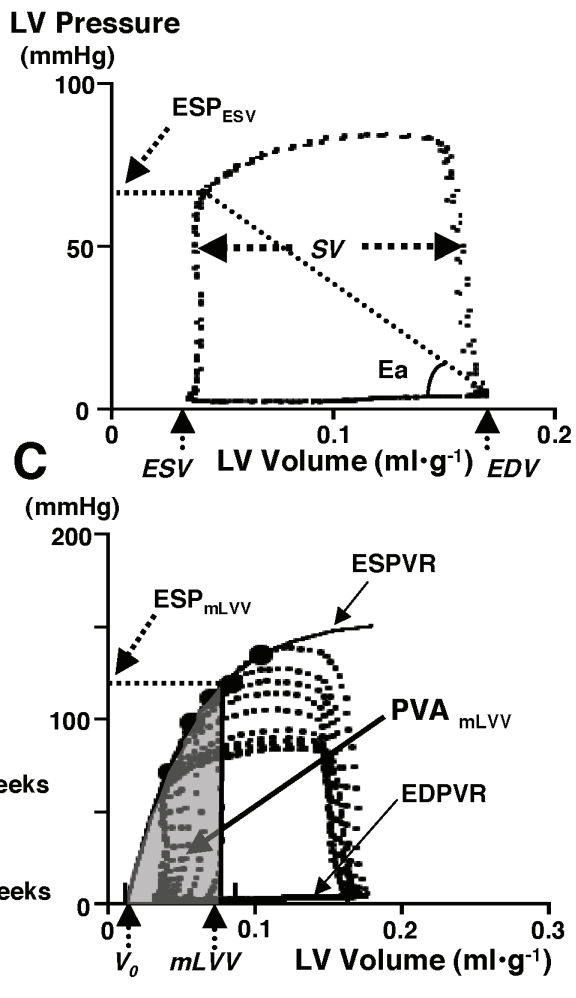

Fig. 1. A: Protocols for the short-term treatment of each rat in (I) Sa, (II) Iso3d, Iso3d(-), and (III) Iso7d, Iso7d(-) groups, and the long-term treatment of each rat in (I) Sa and (II) Iso groups. $\mathrm{LV}$, left ventricle. B: The parameters evaluating for pressurevolume ( $\mathrm{P}-\mathrm{V})$ loop and end-systolic pressure-volume relation (ESPVR). Upper panel: $\mathrm{ESP}_{\mathrm{ESV}}$, end-systolic pressure at end- systolic volume; SV, stroke volume; EDV, end-diastolic volume; ESV, end-systolic volume; Ea, effective arterial elastance defined as $\mathrm{ESP}_{\mathrm{ESV}} / \mathrm{SV}$. Lower panel: $\mathrm{V}_{0}$, volume-intercept of ESPVR; PVA $\mathrm{mLVV}$, systolic pressure-volume area at $\mathrm{mLVV}$ (midrange LVV at end-systole); $\mathrm{ESP}_{\mathrm{mLVV}}$, end-systolic pressure at $\mathrm{mLVV}$. 
the implantation under pentobarbital anesthesia. One hour after removal of the minipump in Sa, Iso $3 \mathrm{~d}$, and Iso $7 \mathrm{~d}$ groups and two days after removal of the minipump in Iso $3 \mathrm{~d}$ and Iso $7 \mathrm{~d}$ groups (Iso $3 \mathrm{~d}[-]$ and Iso $7 \mathrm{~d}[-], n=7$ each), the left ventricular function was evaluated (see protocol shown in Fig. 1A).

Polyacrylamide gel electrophoresis and Western blots for sarcoplasmic reticulum $\mathrm{Ca}^{2+} \mathrm{ATPase}$ (SERCA2), phospholamban (PLB), phospho-Ser ${ }^{16}$ PLB (p-PLB), and $\mathrm{Na}^{+} / \mathrm{Ca}^{2+}$ exchanger (NCX1). Membrane proteins from the left ventricular myocardium of each heart were isolated as described previously $[20,23]$. The frozen hearts were homogenized and centrifuged at $1,000 \times g$ for $10 \mathrm{~min}$. The supernatants were centrifuged at $100,000 \times g$ for $60 \mathrm{~min}$ at $4^{\circ} \mathrm{C}$. The $100,000 \mathrm{~g}$ pellets were cellular membrane fractions and used for immunoblotting of SERCA2, PLB, pPLB, and NCX1.

The same amount of membrane proteins (20 $\mu \mathrm{g} / \mathrm{lane})$ were separated on SDS-polyacrylamide gels (10\% for SERCA2, 15\% for PLB and p-PLB, and 7.5\% for NCX1) in a minigel apparatus (Mini-PROTEAN II, Bio-Rad) and transferred to polyvinyliodene difluoride membranes. The membranes were blocked (4\% Block Ace, Dainippon Pharmaceutical Co., Osaka) and then incubated with antiSERCA2 antibody (1:1,000 dilution, Affinity Bio Reagents), anti-PLB antibody (1:2,000 dilution, Upstate Biotechnology), antiphospho-PLB ( $\left.\mathrm{Ser}^{16}\right)$ antibody (1:1,000 dilution, Upstate Biotechnology), or anti-NCX1 antibody (1:200 dilution, a generous gift from Dr. Takahiro Iwamoto, Fukuoka University). The antigens were detected by the luminescence method (ECL Western blotting detection kit, Amersham) with peroxidase-linked antimouse IgG (1:1,000 dilution). After BEING immunoblotted, the film was scanned, and the intensity of the bands was calculated by NIH image analysis.

Measurements of left ventricular volume and pressure. One hour or two days after the removal of the minipump, we evaluated the left ventricular function in hypertrophied rat hearts by simultaneous measurements of left ventricular volume and left ventricular pressure. The rat was anesthetized with ethylcarbamate $(0.7 \mathrm{~g} / \mathrm{kg}$, i.p.) and $\alpha$-chloralose (60 mg/kg, i.p.). The trachea was intubated, and the rat was ventilated with room air. The chest was opened and a conductance catheter $(1.5 \mathrm{~F})$ was introduced into the left ventricular through an apical stab. A 3.0 F catheter-tip micromanometer was also inserted through the apex into the left ventricle to obtain a reliable left ventricular pressure data.

The principle of the conductance catheter method of measuring left ventricular volume has been described in detail [24-26]. When hemodynamics was stable, a series of left ventricular pressure-volume loops was obtained during increasing afterload by a gradual occlusion of the ascending aorta. The occlusion was performed for 1-2 s until end-diastolic volume slightly increased (almost un- changed), and it was limited so as not to evoke any arrhythmia. Therefore preload recruitable stroke work is not available. This intervention was repeated six times at 10 min intervals. The respirator was stopped during data acquisition to avoid respiratory fluctuation influences on cardiac signals.

In the final part of each experiment, parallel conductance and thus constant offset volume were measured by injecting hypertonic saline $(10 \% \mathrm{NaCl}$ solution; $0.025 \mathrm{ml})$ into the pulmonary artery to transiently change the resistivity of the blood in the left ventricle $[15,16]$. The calculated constant offset volume was subtracted from the measured left ventricular conductance volume to obtain left ventricular absolute blood volume, i.e., absolute left ventricular volume. Left ventricular pressure and the three individual segmental conductance volume signals were digitized and stored in a 12-bit accuracy at a sampling frequency of $500 \mathrm{~Hz}$ for later analyses. At the end of each experiment, a lethal dose of pentobarbital sodium was injected into the rat. The left ventricle, including the interventricular septum, and the right ventricle were excised and weighed, respectively.

Data analysis. In the in situ rat left ventricle, a curvilinear end-systolic pressure-volume relation is obtained by drawing an upper enveloping curve on a series of pressure-volume loops in a manner similar to previously reported methods $[15,16]$. The left ventricular end-systolic pressure-volume data on the left-upper shoulder of all pressure-volume loops were plotted and fitted by the method of least-squares using the following equation, left ventricular pressure $=\mathrm{A}\{1-\exp [-\mathrm{B}$ (left ventricular volume $\left.\left.-\mathrm{V}_{0}\right)\right]$, where $\mathrm{A}, \mathrm{B}$, and $\mathrm{V}_{0}$ are fitted parameters. We obtained the best-fit end-systolic pressure-volume relation curve in each heart in Sa, Iso $3 \mathrm{~d}$, Iso $7 \mathrm{~d}$, Iso $3 \mathrm{~d}(-)$, and Iso $7 \mathrm{~d}(-)$ groups. $\mathrm{V}_{0}$ (systolic unstressed volume) has been previously measured in postmortem isolated rat normal left ventricle. The $\mathrm{V}_{0}$ values are determined to be 0.02 $\pm 0.005 \mathrm{ml} / \mathrm{g}(n=7)$ [17]. In the present study, the mean $\mathrm{V}_{0}$ values of hearts in the Sa group obtained as volume-intercepts of the best-fit end-systolic pressure-volume relations was $0.025 \pm 0.014 \mathrm{ml} / \mathrm{g}(n=7)$ [14]. Therefore we judged that the $\mathrm{V}_{0}$ values obtained by curve-fit are reliable.

Left ventricular PVA is a measure of the total mechanical energy generated by a left ventricular contraction. The PVA of an isovolumic contraction represents the maximal capability of external mechanical work of the left ventricle at a given preload [27]. In the present study, PVA was defined as the area surrounded by the best-fit end-systolic pressure-volume relation curve, the volume axis (instead of negligibly small end-diastolic pressure-volume relationship curve), and the vertical isovolumic pressure-volume line at any preloaded left ventricular volume, such as midrange left ventricular volume (mLVV) (Fig. 1C).

The PVA as a function of the left ventricular volume 
was obtained by integrating the above exponential function from the extrapolated $\mathrm{V}_{0}$ along the volume axis:

$\mathrm{PVA}=\mathrm{A}\left(\right.$ left ventricular volume $\left.-\mathrm{V}_{0}\right)-\mathrm{A}\{1-$ $\exp \left[-\mathrm{B}\left(\right.\right.$ left ventricular volume $\left.\left.\left.-\mathrm{V}_{0}\right)\right]\right\} / \mathrm{B}$.

We had proposed that in in situ hearts, PVA at an appropriate left ventricular volume on the curvilinear PVA-volume relationship is valuable to evaluate left ventricular mechanoenergetics $[15,16]$. In the present study, we calculated midrange left ventricular volume that was the value of $\left[\mathrm{V}_{0}+\right.$ (maximum end-systolic volume - minimum end-systolic volume) on the end-systolic pressure-volume relation $\times 1 / 2$ ] from each pressure-volume loop. Each example of end-systolic pressure and PVA at midrange left ventricular volume $\left(\mathrm{ESP}_{\mathrm{mLVV}}\right.$ and $\left.\mathrm{PVA}_{\mathrm{mLVV}}\right)$ is shown in the lower panel of Fig. 1C. End-diastolic pressure-volume relations are very close to the volume axis, as shown in Fig. 1, B and C, and thus the effect of excluding the measurement of end-diastolic pressure-volume relation would be small. Ea (effective arterial elastance) is defined as the ratio of end-systolic pressure at end-systolic volume (ES$\left.P_{E S V}\right)$ to stroke volume of the left ventricle under stable hemodynamics. Stroke volume was obtained by the formula (end-diastolic volume - end-systolic volume) (Fig. 1B). Ejection fraction was obtained by the formula [stroke volume/(end-diastolic volume $-\mathrm{V}_{0}$ )] .

Echocardiography. For the less-invasive study to follow up the heart function in the same rats (ejection fraction and fractional shortening), the measurements of left ventricular internal dimensions during systole and diastole (LVIDs and LVIDd) and wall thickness (interventricular septum thickness during systole and diastole, IVSTs and IVSTd; left ventricular posterior wall thickness during systole and diastole, LVPWTs and LVPWTd) by tran- sthoracic echocardiography was performed at 0 and 3 days, and $1,2,4,6,8,10,12,14$, and 16 weeks in the Iso $(n=3-8)$ and Sa groups $(n=3)$. Two hours after the removal of an osmotic minipump including Iso or Sa under ethyl ether anesthesia, the same rats were again anesthetized with ethyl ether. An imaging of the heart was done using a 12-MHz ultrasound probe (PLT-1202S) and an echograph, Aplio SSA-700A (Toshiba, Tokyo, Japan). Mmode recordings were performed at the level of papillary muscles. Left ventricular fractional shortening [100 $\times$ (LVIDd - LVIDs)/LVIDd] and ejection fraction $(100 \times$ stroke volume/end-diastolic volume) were analyzed using an installed software program in the echograph. After the recording, an osmotic minipump including Iso or Sa was again implanted. This procedure was repeated throughout the entire 16 weeks.

Statistical analysis. A comparison of paired and unpaired individual values was performed by paired and unpaired $t$-tests, respectively. Multiple comparisons were performed by one-way or repeated measured analysis of variance (ANOVA) with a post hoc Bonferroni's test or the Fisher's PLSD method. A value of $P<0.05$ was considered statistically significant. All data are expressed as the mean $\pm \mathrm{SD}$.

\section{RESULTS}

\section{Cardiac weights (Fig. 2)}

Left ventricular wet weight/body weight was $1.94 \pm$ $0.10 \mathrm{mg} / \mathrm{g}$ in Sa and significantly increased by $35 \%$ in the Iso $3 \mathrm{~d}$ group and by $29 \%$ in the Iso $7 \mathrm{~d}$ group $(P<0.05 \mathrm{vs}$. $\mathrm{Sa})$. The right ventricular wet weight/body weight was $0.541 \pm 0.040 \mathrm{mg} / \mathrm{g}$ in Sa and significantly increased by $22 \%$ in the Iso $3 \mathrm{~d}$ group and $26 \%$ in the Iso $7 \mathrm{~d}$ group $(P<$
A

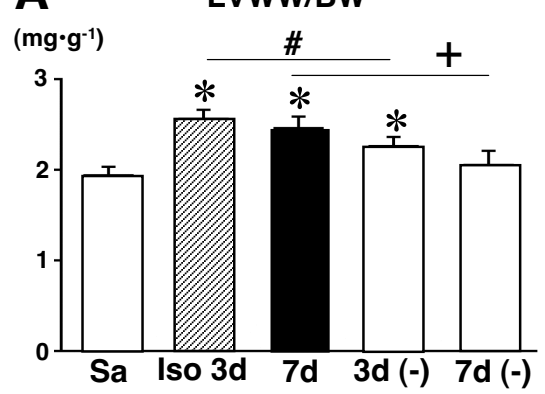

C
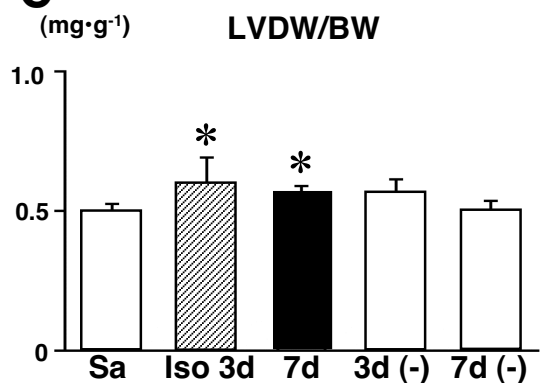

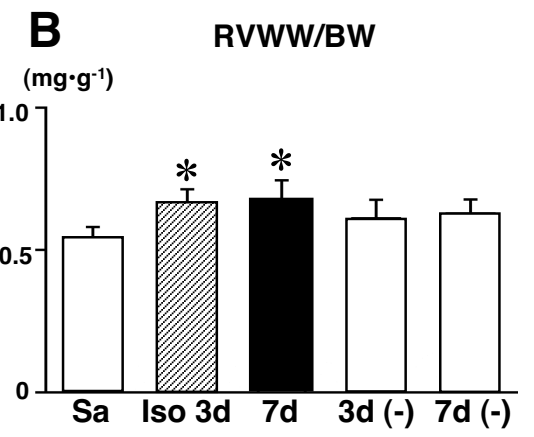

D

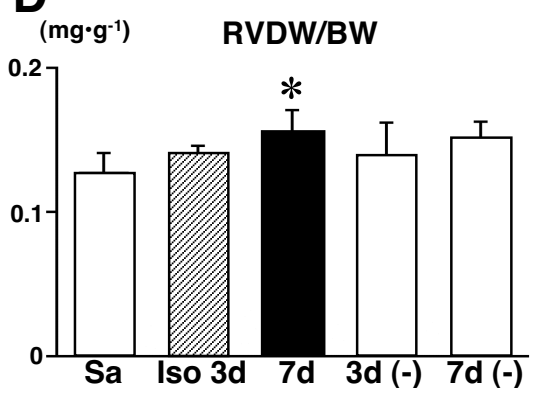

Fig. 2. Comparison of cardiac wet weights and dry weights among Sa, Iso $3 d$, Iso $7 d$, Iso $3 d(-)$, and Iso $7 d(-)$ groups. Sa, saline (24 $\mu$ l/day for 3 days) infused group; Iso $3 d$ or Iso $7 \mathrm{~d}$, isoproterenol $\left(1.2 \mathrm{mg} \cdot \mathrm{kg}^{-1} \cdot \mathrm{day}^{-1}\right.$ for 3 days and 7 days) infused group; Iso $3 \mathrm{~d}(-)$ or iso $7 \mathrm{~d}(-)$ : 2 days after removal of osmotic minipump in isoproterenol (1.2 $\mathrm{mg} \cdot \mathrm{kg}^{-1} \cdot$ day $^{-1}$ for 3 days and 7 days) infused group; BW, body weight; WW, wet weight; DW, dry weight; LV, left ventricle; $\mathrm{RV}$, right ventricle; ${ }^{*} P<0.05$ vs. Sa, ${ }^{\#} P<$ 0.05 vs. Iso $3 \mathrm{~d},{ }^{+} P<0.05$ vs. Iso $7 \mathrm{~d}$. 
0.05 vs. Sa). Left ventricular dry weight/body weight was $0.488 \pm 0.037 \mathrm{mg} / \mathrm{g}$ in Sa and significantly increased by $19 \%$ in the Iso $3 \mathrm{~d}$ group and by $12 \%$ in the Iso $7 \mathrm{~d}$ group $(P$ $<0.05$ vs. Sa). Right ventricular dry weight/body weight was not significantly increased in the Iso $3 \mathrm{~d}$ group, but it increased by $24 \%$ in the Iso $7 d$ group $(P<0.05$ vs. Sa). There were no significant differences in all wet and dry weights/body weight between the Iso $3 \mathrm{~d}$ and Iso $7 \mathrm{~d}$ groups and between the Iso $3 \mathrm{~d}(-)$ and Iso $7 \mathrm{~d}(-)$ groups.

\section{Western blot analyses}

Each amount of expression of SERCA2 and PLB was significantly decreased in the Iso $3 \mathrm{~d}$ group $(P<0.05)$ from that in the Sa group and returned to almost the same level in the Iso 7d group as in the Sa group (Fig. 3). Consequently, the ratio of expression of SERCA2/PLB was unchanged in all three groups. However, each amount of expression of phospho-Ser ${ }^{16}$ PLB in the Iso3d and Iso7d groups was significantly smaller than that in the Sa group. Furthermore, the amount of expression of NCX1 was also unchanged in all three groups (Fig. 3). The amount of expression of SERCA2 and PLB and the ratio of expression of SERCA2 versus PLB in the Iso $3 \mathrm{~d}(-)$ and Iso $7 \mathrm{~d}(-)$ groups was almost at the same level as those in the $\mathrm{Sa}$ group (data not shown).

\section{Comparison of pressure-volume loops and end- systolic pressure-volume relations among $\mathrm{Sa}$, Iso $3 \mathrm{~d}$, Iso $7 \mathrm{~d}$, Iso $3 \mathrm{~d}(-)$, and Iso $7 \mathrm{~d}(-)$ groups}

Representative sets of pressure-volume loops for a typical heart from all five groups (Fig. 4). The shapes of endsystolic pressure-volume relations hardly differed among Sa, Iso $3 \mathrm{~d}$, and Iso $7 \mathrm{~d}$ groups. The working ranges of left ventricular volume were markedly different in hypertro- phied hearts in both the Iso $3 \mathrm{~d}$ and Iso $7 \mathrm{~d}$ groups from those in the Sa group (Fig. 4, B and C, vs. A). Respective hearts from Iso $3 \mathrm{~d}(-)$ and Iso $7 \mathrm{~d}(-)$ groups showed pressure-volume loops and end-systolic pressure-volume relations almost similar to those in a heart from the Sa group (Fig. 4, D and E, vs. A).

\section{Mean midrange left ventricular volume (mLVV), $\mathrm{ESP}_{\mathrm{mLVV}}$, and PVA $\mathrm{mLVV}$}

Mean mLVV in the Iso $3 \mathrm{~d}$ ( $45 \%$ of $\mathrm{Sa})$ and the Iso $7 \mathrm{~d}$ group (61\% of $\mathrm{Sa}$ ) was significantly smaller than in the $\mathrm{Sa}$ group $(0.123 \pm 0.032 \mathrm{ml} / \mathrm{g})(P<0.05)$, but mean $\mathrm{mLVV}$ in the Iso $3 \mathrm{~d}(-)$ and Iso $7 \mathrm{~d}(-)$ groups was not significantly different from that in the Sa group. There were no significant differences in mean mLVV between the Iso $3 \mathrm{~d}$ and Iso $7 \mathrm{~d}$ groups or between the Iso $3 \mathrm{~d}(-)$ and Iso $7 \mathrm{~d}(-)$ groups (Fig. 5A).

Mean $\mathrm{ESP}_{\mathrm{mLVV}}$ in the Iso $3 \mathrm{~d}$ and Iso $7 \mathrm{~d}$ groups was not significantly different from that in $\mathrm{Sa}$, and there were no significant differences between the Iso $3 \mathrm{~d}$ and Iso $7 \mathrm{~d}$ groups or the Iso $3 \mathrm{~d}(-)$ and Iso $7 \mathrm{~d}(-)$ groups (Fig. 5B).

However, mean $\mathrm{PVA}_{\mathrm{mLVV}}$ in the Iso $3 \mathrm{~d}(49 \%$ of $\mathrm{Sa})$ and the Iso $7 d$ group ( $49 \%$ of $\mathrm{Sa}$ ) was significantly smaller than that in the Sa group $\left(7.16 \pm 2.72 \mathrm{mmHg} \cdot \mathrm{ml} \cdot\right.$ beat $\left.^{-1} \cdot \mathrm{g}^{-1}\right)$ $(P<0.05)$, although there were no significant differences in $\mathrm{PVA}_{\mathrm{mLVV}}$ between the Iso $3 \mathrm{~d}$ and Iso $7 \mathrm{~d}$ groups (Fig. 5C). Mean PVA ${ }_{m L V V}$ in the Iso $3 \mathrm{~d}(-)$ and Iso $7 \mathrm{~d}(-)$ groups was not significantly different from that in the Sa group, and there were no significant differences between the Iso $3 \mathrm{~d}(-)$ and Iso $7 \mathrm{~d}(-)$ groups (Fig. 5C). The impairment of the left ventricular mechanical work capability in the Iso $3 \mathrm{~d}$ and Iso $7 \mathrm{~d}$ groups and its reversibility by the removal of Iso seem to be similar.

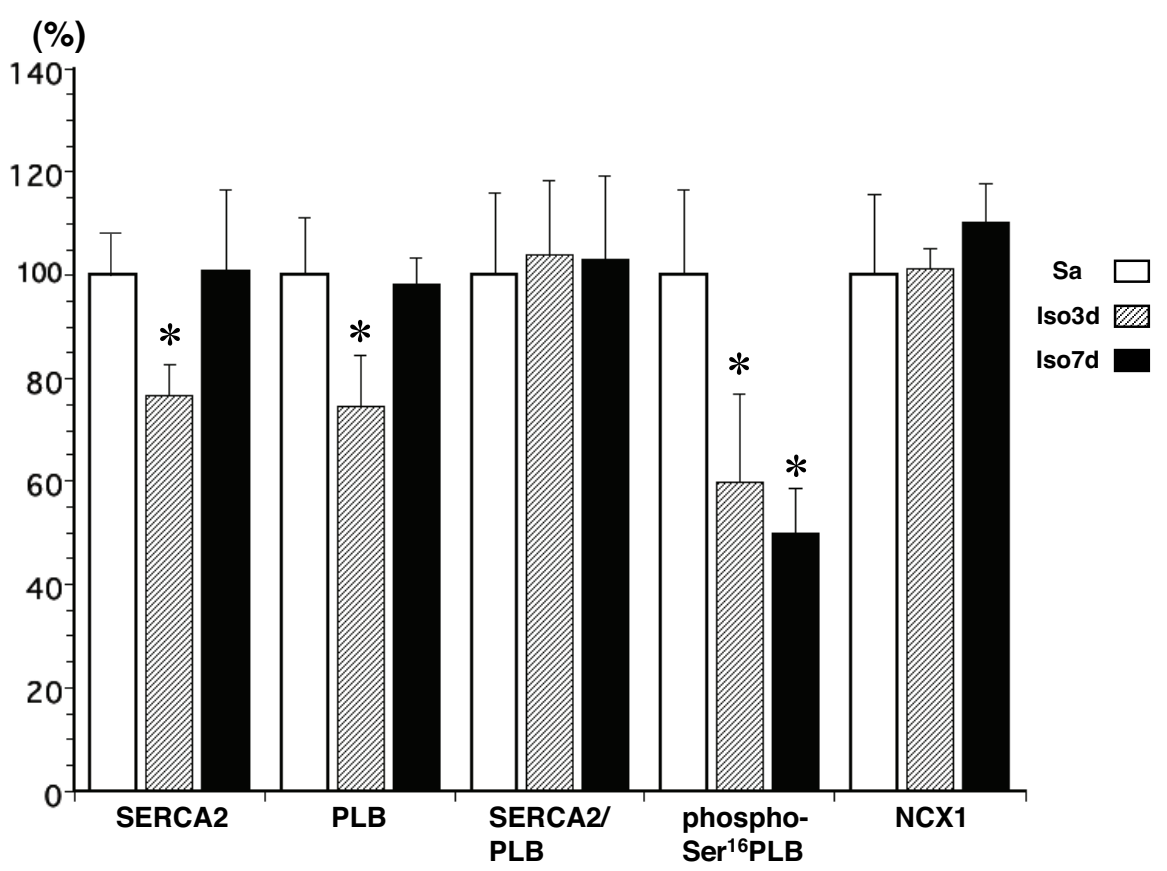

Fig. 3. Comparison of Western blots of SERCA2, PLB, SERCA2/PLB, phospho-Ser ${ }^{16}$ PLB, NCX1 in each heart among Sa, Iso3d, and Iso7d groups. ${ }^{*} P$ $<0.05$ vs. Sa group. 

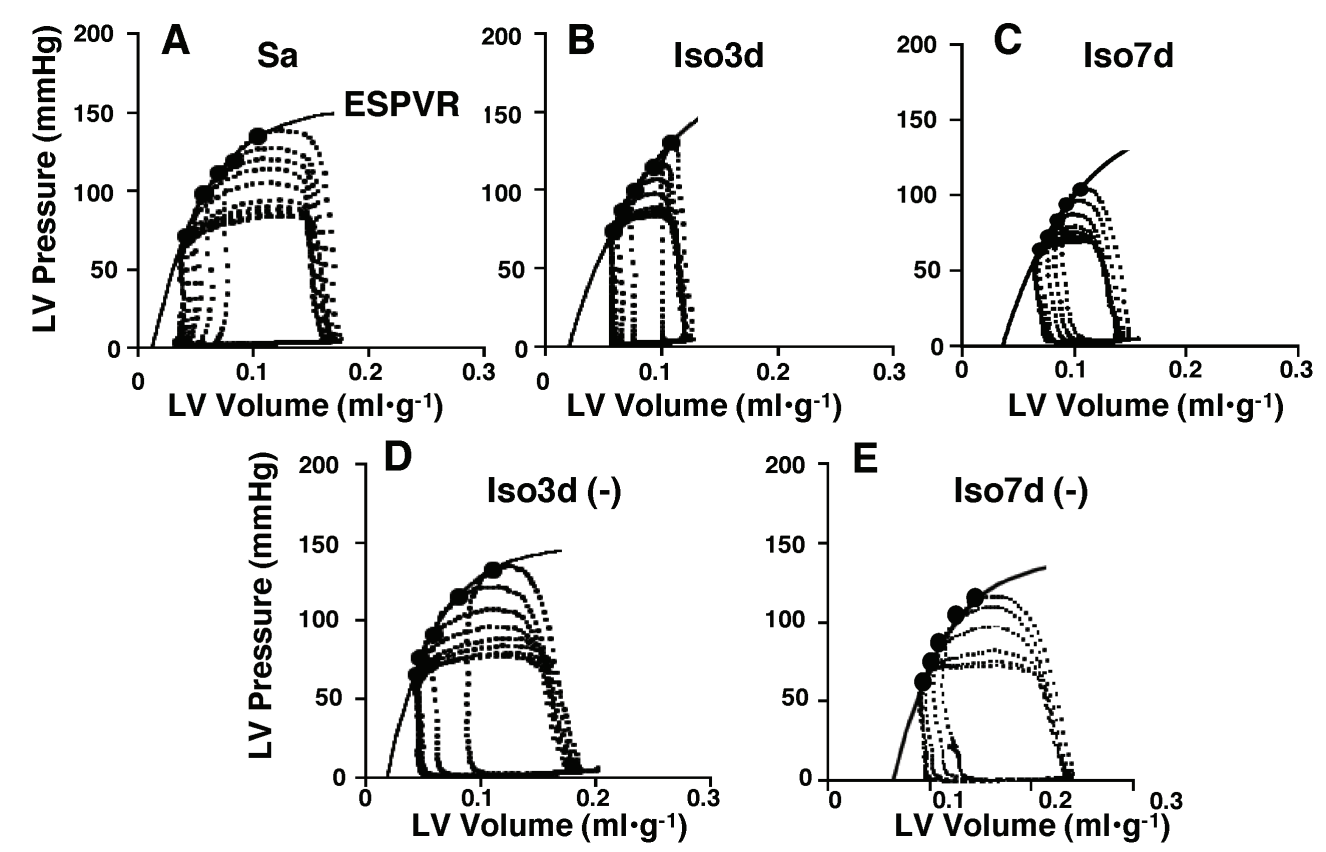

Fig. 4. Each representative set of $\mathrm{P}-\mathrm{V}$ loops and ESPVR in Sa (A), Iso $3 d(B)$, Iso $7 d(\mathbf{C})$, Iso $3 d(-)(D)$, and Iso $7 d(-)(E)$.
A
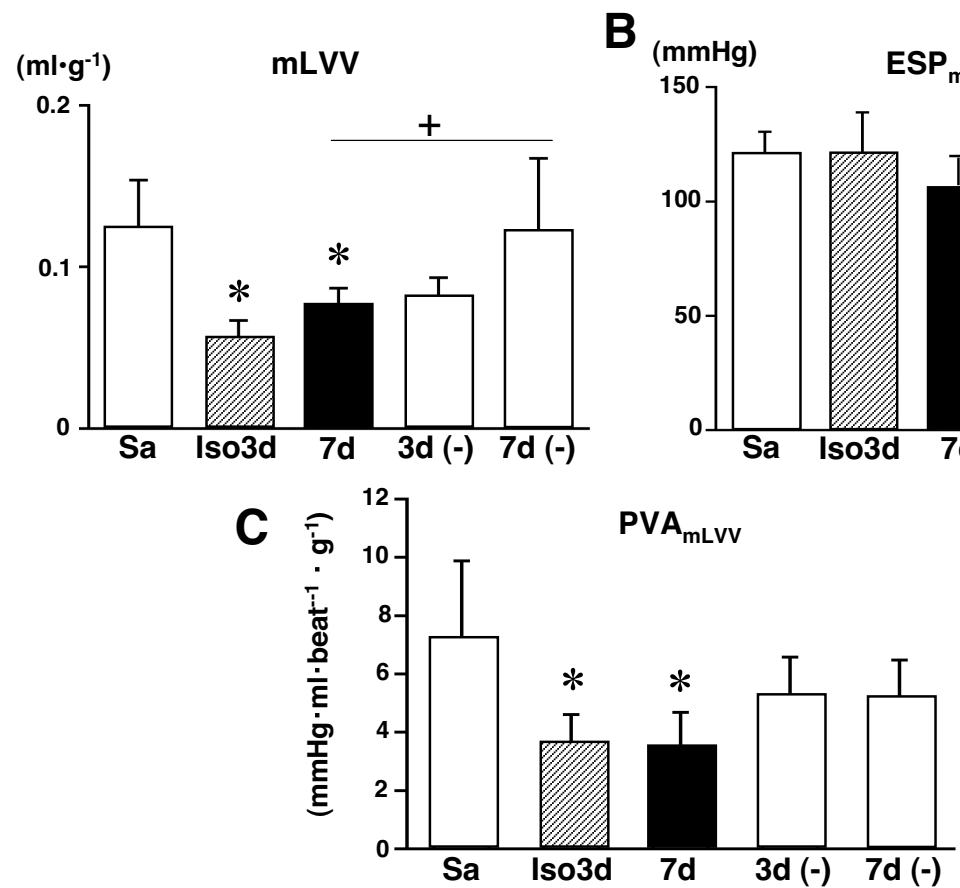

Fig. 5. Mean $\mathrm{mLV}$ (midrange LVV at end-systole) (A), ESP $\mathrm{mLW}_{\mathrm{m}}$ (B), and PVA $\mathrm{mLVV}(\mathbf{C})$ in Sa, Iso $3 d$, Iso $7 d$, Iso $3 d(-)$, and Iso $7 \mathrm{~d}(-)$ groups. ${ }^{*} P<0.05$ vs. Sa. $+P<0.05$ vs. Iso $7 \mathrm{~d}$.

\section{Other hemodynamic parameters}

Mean end-diastolic volume, stroke volume, and cardiac output in the Iso $3 \mathrm{~d}$ and Iso $7 \mathrm{~d}$ groups were significantly smaller than those in the Sa group $(P<0.05)$ (Fig. 6), but mean ejection fraction and mean $\mathrm{ESP}_{\mathrm{ESV}}$ were unchanged in all groups (Table 1). Mean Ea was significantly larger in Iso $3 \mathrm{~d}(175 \%$ of $\mathrm{Sa})$ because of smaller stroke volume, but not in the other three groups (Table 1). Mean end-systolic volume, end-diastolic volume, stroke volume, cardiac output, ejection fraction, $\mathrm{ESP}_{\mathrm{ESV}}$, and $\mathrm{Ea}$ in the Iso $3 \mathrm{~d}(-)$ and Iso $7 \mathrm{~d}(-)$ groups did not differ from those in the Sa group. All hemodynamic parameters were not significantly different between the Iso $3 \mathrm{~d}$ and Iso $7 \mathrm{~d}$ groups or between the Iso $3 \mathrm{~d}(-)$ and Iso $7 \mathrm{~d}(-)$ groups (Fig. 6 and Table 1). However, in ESV and EDV, there were significant differences between the Iso $7 \mathrm{~d}$ and Iso $7 \mathrm{~d}(-)$ groups, whereas there were no significant differences between the Iso $3 \mathrm{~d}$ and Iso $3 \mathrm{~d}(-)$ groups (Fig. 6). Although it appeared that the reversibility from Iso $7 \mathrm{~d}$ by the removal of Iso was better than that from Iso $3 \mathrm{~d}$ by its removal, this may be due to marked increases and fluctuations in $\mathrm{V}_{0}$ values in the Iso $7 \mathrm{~d}(-)$ group (Table 1$)$. In fact, the reversibility of SV and CO in Iso $3 \mathrm{~d}(-)$ was significant, but not in Iso $7 \mathrm{~d}(-)$, as predicted (Fig. 6).

Although mean heart rate was significantly smaller in the Iso $3 \mathrm{~d}$ group $(P<0.05)$, in other groups it did not differ 
A

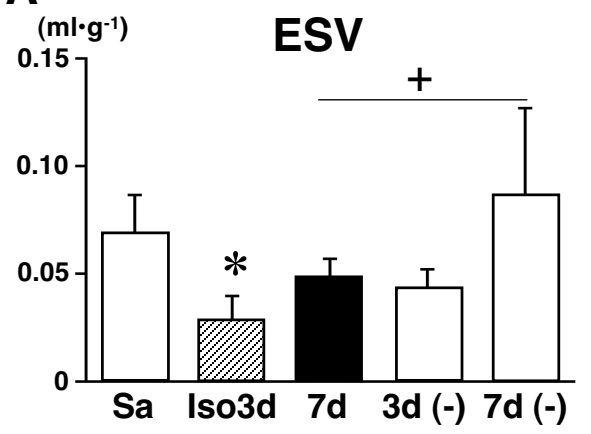

C

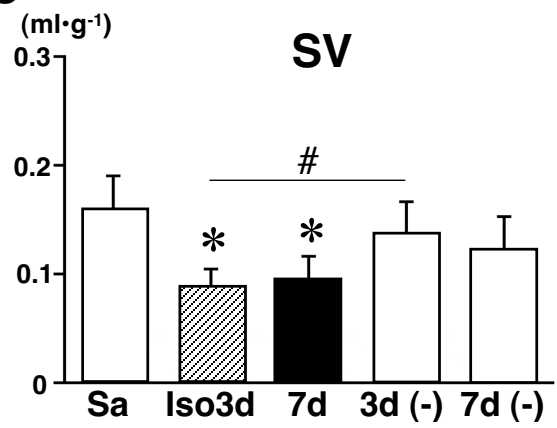

B

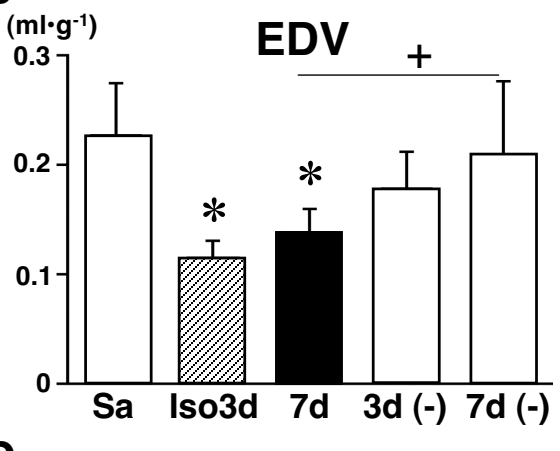

D

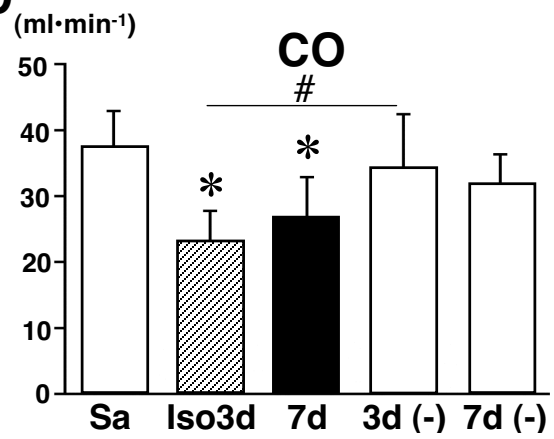

Fig. 6. Mean ESV (end-systolic volume) (A), EDV (end-diastolic volume) (B), SV (stroke volume) (C), and CO (cardiac output) (D) in Sa, Iso $3 d$, Iso $7 \mathrm{~d}$, Iso $3 \mathrm{~d}(-)$, and Iso $7 \mathrm{~d}(-)$ groups. ${ }^{*} P$ $<0.05$ vs. Sa, ${ }^{\#} P<0.05$ vs. Iso $3 \mathrm{~d},{ }^{+} P<$ 0.05 vs. Iso $7 \mathrm{~d}$.

Table 1. Hemodynamics based on in situ analysis of pressure-volume loops.

\begin{tabular}{|c|c|c|c|c|c|}
\hline$n$ & $\begin{array}{l}\mathrm{Sa} \\
(7)\end{array}$ & $\begin{array}{c}\text { Iso } 3 d \\
(7)\end{array}$ & $\begin{array}{c}\text { Iso } 7 d \\
(7)\end{array}$ & $\begin{array}{c}\text { Iso } 3 d(-) \\
(7)\end{array}$ & $\begin{array}{c}\text { Iso } 7 d(-) \\
(7)\end{array}$ \\
\hline $\mathrm{V}_{0}(\mathrm{ml} / \mathrm{g})$ & $0.025 \pm 0.014$ & $0.008 \pm 0.005^{\star}$ & $0.014 \pm 0.012$ & $0.010 \pm 0.006^{*}$ & $0.030 \pm 0.036$ \\
\hline EF (\%) & $70.6 \pm 4.1$ & $75.7 \pm 9.9$ & $66.2 \pm 9.7$ & $76.1 \pm 1.5$ & $60.0 \pm 7.9$ \\
\hline $\mathrm{ESP}_{\mathrm{ESV}}(\mathrm{mmHg})$ & $71 \pm 7$ & $70 \pm 5$ & $66 \pm 2$ & $78 \pm 7$ & $72 \pm 5$ \\
\hline $\mathrm{Ea}\left(\mathrm{mmHg} \cdot \mathrm{ml}^{-1} \cdot \mathrm{g}\right)$ & $482 \pm 128$ & $843 \pm 181^{*}$ & $769 \pm 222$ & $607 \pm 169$ & $618 \pm 153$ \\
\hline HR (beats/min) & $354 \pm 28$ & $293 \pm 16^{*}$ & $308 \pm 26$ & $329 \pm 54$ & $327 \pm 30$ \\
\hline
\end{tabular}

$\mathrm{V}_{0}$, systolic unstressed volume; $\mathrm{ESP}_{\mathrm{ESV}}$, end-systolic pressure at $\mathrm{ESV} ; \mathrm{ESP}_{\mathrm{mLV}}$, end-systolic pressure at $\mathrm{mLVV}$ (midrange LVV); Ea, Effective arterial elastance; HR, heart rate. ${ }^{\star} P<0.05$ vs. Sa.

from that in the Sa group (Table 1). We have previously reported that the curved end-systolic pressure-volume relation is independent of heart rate in in situ rat hearts within 250-320 bpm [16] and in excised rat hearts within 250$300 \mathrm{bpm}$ [21]. In the present study, the mean heart rate distributed from 293-354 bpm, indicating that the curved end-systolic pressure-volume relation will not be affected by heart rate.

\section{Echocardiographic evaluation of heart function and wall thickness}

At 3 days and at 1 and 2 weeks, ejection fraction and fractional shortening in the Iso-infused rat group was not significantly decreased from those in the Sa-infused group. At 4 weeks, the ejection fraction was decreased by $12 \%$, and this was accompanied by a $25 \%$ decrease in fractional shortening from those at day 0 in the Iso-infused rat group. These values at 4 weeks were significantly $(P<0.05)$ smaller than the respective data in the Sa-in- fused rat group (Fig. 7A). In the 6-14-week Iso-infused rat group, both ejection fraction and fractional shortening did not further decrease, though both decreases remained significant $(P<0.05)$ compared with the respective data in the Sa-infused rat group (Fig. 7A).

At 4 weeks, left ventricular internal dimensions during systole (LVIDs) were increased by $43 \%$ from those at day 0 in the Iso-infused rat group, and this increased value was significantly $(P<0.05)$ larger and remained significant $(P$ $<0.05)$ through the 16 weeks compared with respective data in the Sa-infused rat group (Fig. 7B). In contrast, left ventricular internal dimensions during diastole (LVIDd) in the Iso-infused rat group was not significantly larger than those in the Sa-infused rat group throughout the entire 16 weeks (Fig. 7B).

At 3 days and 1 week, interventricular septum thickness during diastole (IVSTd) as well as left ventricular posterior wall thickness during diastole (LVPWTd) were significantly $(P<0.05)$ increased by $20 \%-34 \%$ from 
those at day 0 in Iso-infused rat hearts, and their increases remained significant $(P<0.05)$ throughout the 16 weeks, compared with the respective data in the Sa-infused rat group (Fig. 7C).

Compared with echocardiographic images of Sa-infused rat hearts at 2 and 4 weeks, there was a marked increase in LVIDs at 4 weeks accompanied with already increased LVPWTd and IVSTd at 3 days in Iso-infused rat hearts (Fig. 8), indicating LV systolic dysfunction with hypertrophy.

\section{DISCUSSION}

Although many studies on mechanisms underlying Iso-induced cardiac hypertrophy have been reported [4-13], the conclusive mechanisms have not yet been determined. However, we have revealed the most upstream mechanism: the $\beta_{1}-\mathrm{AR}-$ mediated signal transduction pathway is primarily responsible for all events related to cardiac hypertrophy induced by Iso infusion [14].

The $\beta_{1}$-adrenergic receptors $\left(\beta_{1} \mathrm{ARs}\right)$, which bind circulating catecholamines, activate adenylate cyclase by coupling through the stimulatory G-protein, Gas. The resulting increase in intracellular cAMP activates cAMPdependent protein kinase (PKA). PKA has several pathways in cardiomyocytes that influence contractility in response to activated $\beta_{1}$ AR signaling; these include the Ltype $\mathrm{Ca}^{2+}$ channel in the sarcolemma, the ryanodine re-

Fig. 7. Echocardiographic parameters of heart function at 3 days (D) and 1-16 weeks (WK) in isoproterenol (Iso)-infused rats and saline (Sa)-infused rats. A: EF (ejection fraction) and FS (fractional shortening). B: LVIDs (left ventricular internal dimensions during systole) and LVIDd (left ventricular internal dimensions during diastole). C: IVSTs (interventricular septum thickness during systole) and IVSTd (interventricular septum thickness during diastole). ${ }^{*} P<0.05$ vs. Sa, ${ }^{\#} P<0.05$ vs. 0 WK. ceptor (RyR2), and phospholamban (PLB) in the sarcoplasmic reticulum (SR). The phosphorylation of PLB leads to an increase of SR $\mathrm{Ca}^{2+}$ ATPase (SERCA) activity and consequent accelerated $\mathrm{Ca}^{2+}$ accumulation in the SR. PKA hyperphosphorylation of the RyR2 is observed in
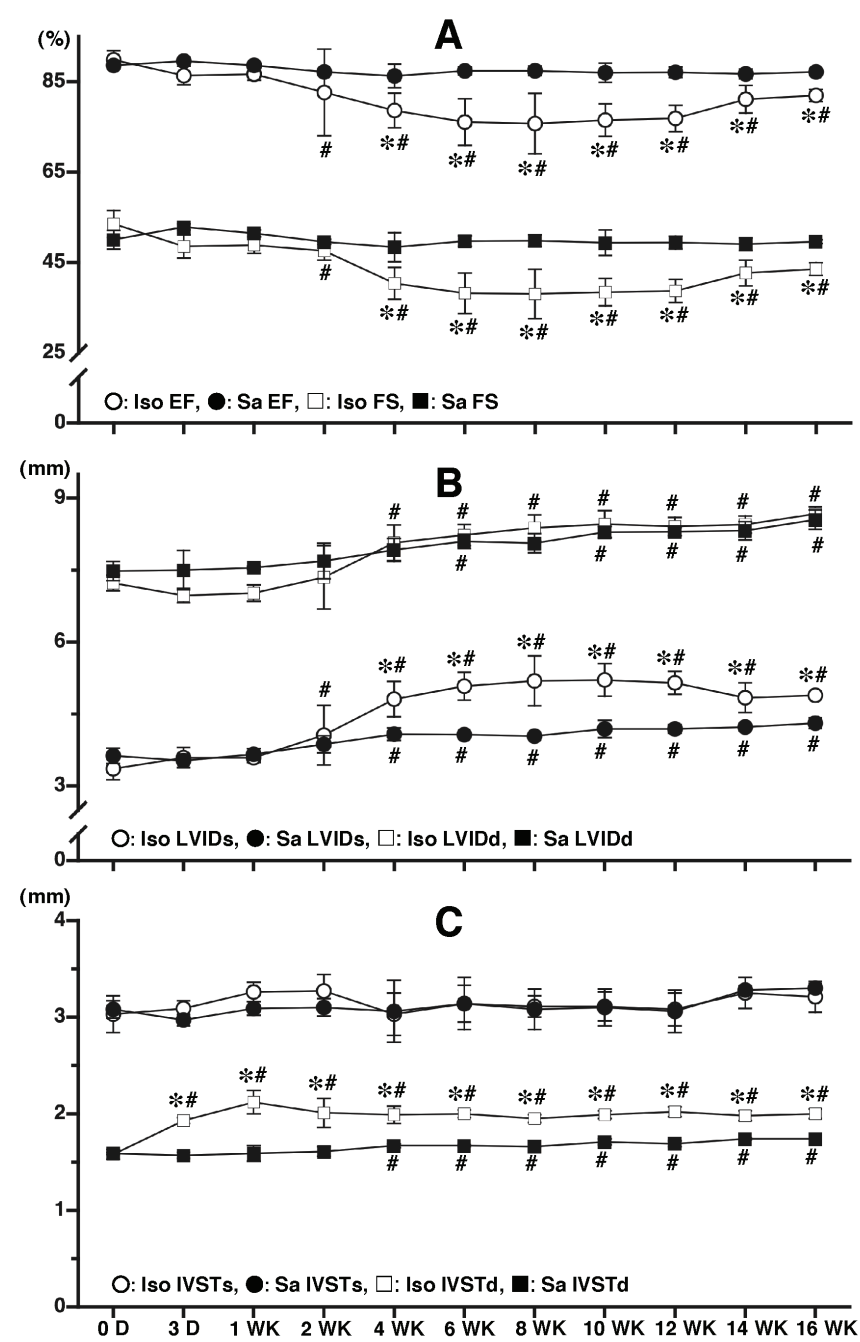

Fig. 8. Echocardiographic images of hearts from saline (Sa)- and isoproterenol (Iso)-infused rats at 2 and 4 weeks. LVIDs, left ventricular internal dimensions during systole; LVIDd, left ventricular internal dimensions during diastole; LVPWTd, left ventricular posterior wall thickness during diastole. is , large LVIDs indicated systolic failure.
2 weeks

4 weeks

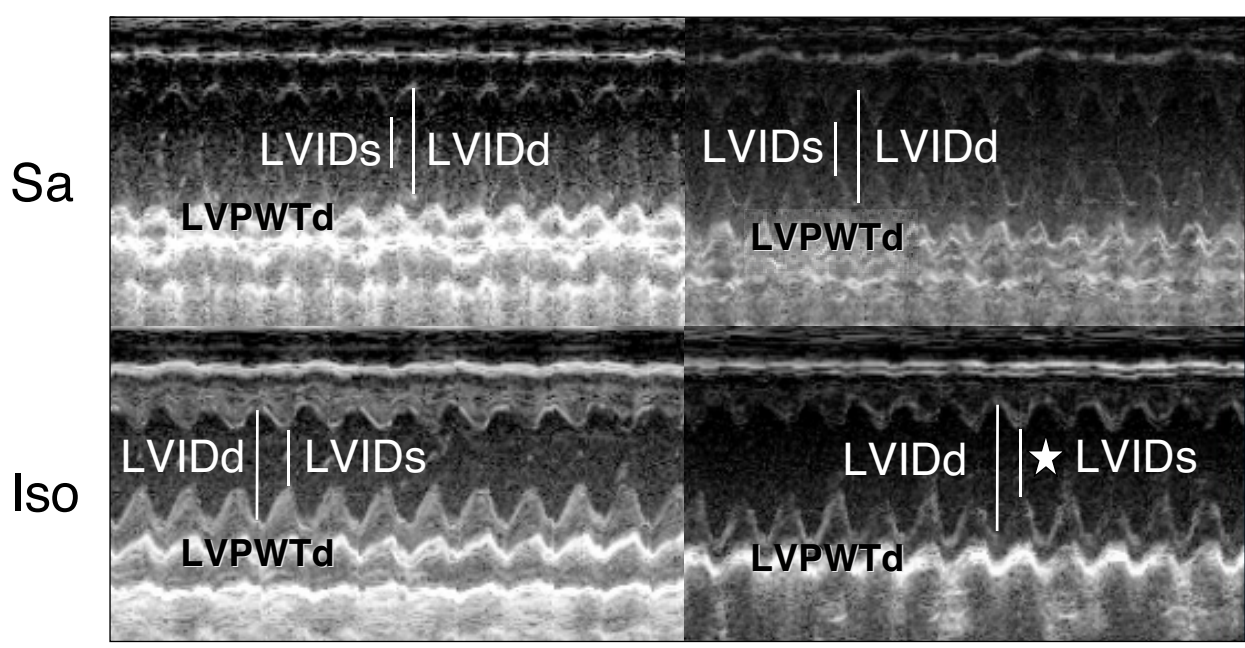


failing human hearts [28], suggesting a link between PKA signaling and negative inotropism associated with longterm $\beta_{1} \mathrm{AR}$ signaling. In the present Iso group at longer than 4 weeks, ejection fraction and fractional shortening actually decreased, accompanied by increased LVIDs, IVSTd, and LVPWTd, indicating systolic failure with hypertrophy (see Figs. 7 and 8).

On the other hand, each amount of expression of phospho-Ser ${ }^{16}$ PLB in the Iso $3 \mathrm{~d}$ and Iso $7 \mathrm{~d}$ groups with shortterm $\beta_{1}$ AR signaling was significantly smaller than that in the Sa group (see Fig. 3). Therefore we predicted diastolic dysfunction because of the impaired $\mathrm{Ca}^{2+}$ uptake function into SR in both groups under the unchanged expression of NCX1 protein presented in Fig. 3. However, we did not observe a marked upward shift of end-diastolic pressurevolume relation corresponding to diastolic dysfunction in the present study, though we found in vitro a left ventricular lusitropic dysfunction in the same hypertrophy model [29]. This might be a limitation of the present pressurevolume measurement system.

Present results suggested that short-term $\beta_{1}$ AR signaling elicited some remodeling of $\mathrm{Ca}^{2+}$ handling in cardiac excitation-contraction coupling, such as the impairment of the $\mathrm{Ca}^{2+}$ uptake function into SR with simultaneous hypertrophy development. Unlike the present hypertrophy model, in the spontaneously hypertensive rats the high afterload may induce some remodeling of $\mathrm{Ca}^{2+}$ handling in cardiac excitation-contraction coupling prior to the development of hypertrophy [30]. The contribution of the remodeling of $\mathrm{Ca}^{2+}$ handling in cardiac excitation-contraction coupling for developing cardiac hypertrophy may not be uniform in respective hypertrophy models.

The simultaneous measurement of left ventricular pressure-volume signals and analysis using the framework of the systolic pressure-volume area (PVA), especially $\mathrm{PVA}_{\mathrm{mLVV}}$, are substantially needed to evaluate the left ventricular function in the heart, because $\mathrm{PVA}_{\mathrm{mLVV}}$ precisely reflects the changes in left ventricular mechanical work capability at mLVV [15-17]. Considering similar decreases in PVA ${ }_{\mathrm{mLVV}}$ of Iso $3 \mathrm{~d}$ and $7 \mathrm{~d}$ groups, we judged that the left ventricular mechanical work capability was impaired in a similar degree in both groups, though no decreases in ejection fraction or fractional shortening were detected.

At 3 days and 1 week in Iso-infused rat data, the wall thickness parameters such as IVSTd and LVPWTd were significantly increased from those at 0 days. This result is in accordance with the significant increase in LV weights and the significant decrease in stroke volume at 3 days and 1 week in Iso-induced rat data (see Figs. 2 and 6). These increases in IVSTd (and LVPWTd) remained significant compared with their respective Sa-infused rat data for up to 16 weeks (see Fig. 7C), indicating that the left ventricular wall thickness never decreased throughout that time.

Although the mean ejection fraction values calculated from a pressure-volume loop were smaller than those calculated by echocardiography, there were no significant differences in mean ejection fraction among the Sa, Iso 3d, and Iso 7d groups (see Table 1). Furthermore, fractional shortening and ejection fraction in Iso-infused rats at 3 days and at 1 and 2 weeks calculated by echocardiography were not significantly decreased from the respective data in Sa-infused rats. These results suggested that after $3 \mathrm{~d}-$, 7d-, and 2-week Iso infusions, marked hemodynamic changes evaluated by echocardiography did not occur. At 4-16 weeks, the fractional shortening and ejection fraction were significantly decreased, accompanied by significantly increased LVIDs compared to those in Sa-infused rats (see Figs. 7 and 8). Taken together, it was suggested that Iso treatment for a term longer than 4 weeks induced systolic dysfunction in a thick-walled heart (hypertrophy), but not the dilated cardiomyopathy in a thin-walled heart, for up to 16 weeks.

The present result indicated that the hypertrophy model was not so markedly changed in function and shape between 3-day and 2-week Iso infusions. During the same term of Iso infusion, expressions of immediate early genes, GATA-4 and Nkx-2.5, Egr-1,c-jun, and c-fos were persistently increased, though after a much higher dose of Iso infusion $\left(30 \mathrm{mg} \cdot \mathrm{kg}^{-1} \cdot \mathrm{day}^{-1}\right)$ than the present one $(1.2$ $\left.\mathrm{mg} \cdot \mathrm{kg}^{-1} \cdot \mathrm{day}^{-1}\right)$ [13]. We concluded that even the shortterm Iso-infused model sufficiently represents the effects of up to a 2-week Iso infusion; it is plausible that the 3-day and 7-day models are corresponding to the 2-week hypertrophy model.

This work was supported in part by Grants-in-Aid for Scientific Research 17390231, 18650125, 20300162 and for Encouragement of Young Scientists 14770016 from the Ministry of Education, Culture, Sports, Science and Technology of Japan. The authors express their thanks to Dr. T. Iwamoto, Fukuoka University, for a generous gift of anti-NCX1 antibody.

\section{REFERENCES}

1. Boluyt MO, Long X, Eschenhagen T, Mende U, Schmitz W, Crow MT, Lakatta EG. Isoproterenol infusion induces alterations in expression of hypertrophyassociated genes in rat heart. Am J Physiol Heart Circ Physiol. 1995;269:H63847.

2. Stanton HC, Brenner G, Mayfield ED Jr. Studies on isoproterenol-induced cardiomegaly in rats. Am Heart J. 1969;77:72-80.

3. Zierhut W, Zimmer HG. Significance of myocardial alpha- and betaadrenoceptors in catecholamine-induced cardiac hypertrophy. Circ Res. 1989;65:1417-25.

4. Zhang G-X, Kimura S, Nishiyama A, Shokoji T, Rahmana M, Yao L, Nagai Y, Fujisawa Y, Miyatake A, Abe Y. Cardiac oxidative stress in acute and chronic isoproterenol-infused rats. Cardiovasc Res. 2005;65:230-8.

5. Zhang G-X, Ohmori K, Nagai Y, Fujisawa Y, Nishiyama A, Abe Y, Kimura S. Role of AT1 receptor in isoproternol-induced cardiac hypertrophy and oxidative stress in mice. J Mol Cell Cardiol. 2007;42:804-11.

6. Kitagawa Y, Tamura Y, Shimizu J, Nakajima-Takenaka C, Taniguchi S, Uesato S, Takaki M. Effects of novel histone deacetylase inhibitor, $\mathrm{N}$-(2-aminophenyl) benzamide on a reversible hypertrophy induced by isoproterenol in in situ rat hearts. J Pharmacol Sci. 2007;104:167-75.

7. Antos CL, Frey N, Marx SO, Reiken S, Gaburjakova M, Richardson JA, Marks $A R$, Olson EN. Dilated cardiomyopathy and sudden death resulting from constitutive activation of protein kinase A. Circ Res. 2001;89:938-40.

8. Leenen FH, White R, Yuan B. Isoproterenol-induced cardiac hypertrophy: role of 


\section{TAKESHITA et al.}

circulatory versus cardiac renin-angiotensin system. Am J Physiol Heart Circ Physiol. 2001;281:H2410-H16.

9. Morisco C, Zebrowski DC, Vatner DE, Vatner SF, Sadoshima J. Beta-adrenergic cardiac hypertrophy is mediated primarily by the beta(1)-subtype in the rat heart. Mol Cell Cardiol. 2001;33:561-573.

10. Nagano M, Higaki J, Nakamura F, Higashimori K, Nagano N, Mikami H, Ogihara T. Role of cardiac angiotensin II in isoproterenol-induced left ventricular hypertrophy. Hypertension. 1992;19:708-12.

11. Sugden PH. Signaling in myocardial hypertrophy: life after calcineurin? Circ Res. 1999;84:633-46

12. Sussman MA, Lim HW, Gude N, Taigen T, Olson EN, Robbins J, Colbert MC, Gualberto A, Wieczorek DF, Molkentin JD. Prevention of cardiac hypertrophy in mice by calcineurin inhibition. Science. 1998;281:1690-93.

13. Saadane N, Apert L, Chalifour LE. Expression of immediate early genes, GATA4 , and Nkx-2.5 in adrenergic-induced cardiac hypertrophy and during regression in adult mice. Br J Pharmacol. 1999;127: 165-76.

14. Kitagawa $Y$, Yamashita D, Ito H, Takaki M. The reversible effects of isoproterenolinduced hypertrophy on in situ left ventricular function in rat hearts. Am J Physiol Heart Circ Physiol. 2004;287:H277-H85.

15. Kishi T, Nakahashi K, Ito H, Taniguchi S, Takaki M. Effects of milrinone on left ventricular end-systolic pressure-volume relationship of rat hearts in situ. Clin Exp Pharmacol Physiol. 2001;28:737-42.

16. Lee S, Ohga Y, Tachibana H, Syuu Y, Ito H, Harada M, Suga H, Takaki M. Effects of myosin isozyme shift on curvilinearity of the left ventricular end-systolic pressure-volume relation of In situ rat hearts. Jpn J Physiol. 1998;48:445-55.

17. Tachibana $\mathrm{H}$, Takaki M, Lee $\mathrm{S}$, Ito $\mathrm{H}$, Yamaguchi $\mathrm{H}$, Suga $\mathrm{H}$. New mechanoenergetic evaluation of left ventricular contractility in in situ rat hearts. Am J Physiol Heart Circ Physiol. 1997;272:H2671-H78.

18. Georgakopoulos D, Kass DA. Minimal force-frequency modulation of inotropy and relaxation of in situ murine heart. J Physiol (Lond). 2001;534:535-45.

19. Kameyama T, Chen Z, Bell SP, Fabian J, LeWinter MM. Mechanoenergetic studies in isolated mouse hearts. Am J Physiol Heart Circ Physiol. 1998;274:H366-74.

20. Ohga Y, Sakata S, Takenaka C, Abe T, Tsuji T, Taniguchi S, Takaki M. Cardiac dysfunction in terms of left ventricular mechanical work and energetics in hypothyroid rats. Am J Physiol Heart Circ Physiol. 2002;283:H631-41.

21. Sakata S, Ohga Y, Abe T, Tabayashi N, Kobayashi S, Tsuji T, Kohzuki H, Misawa $\mathrm{H}$, Taniguchi S, Takaki M. No dependency of a new index for oxygen cost of left ventricular contractility on heart rates in the blood-perfused excised rat hearts. Jpn J Physiol. 2001;51:177-85.

22. Takaki M. Left ventricular mechanoenergetics in small animals. Jpn J Physiol. 2004;54:175-207.

23. Yoshida K, Harada K. Proteolysis of erythrocyte-type and brain-type ankyrins in rat heart after postischemic reperfusion. J Biochem. 1997;122:279-85.

24. Baan J, Aouw Jong TT, Kerkhof PLM, Moene RJ, van Dijk AD, van der Velde ET, Koops J. Continuous stroke volume and cardiac output from intra-ventricular dimensions obtained with impedance catheter. Cardiovasc Res. 1981;15:32834.

25. Baan J, van der Velde ET, De Bruin HG, Smeenk GJ, Koops J, van Dijk AD, Temmerman D, Senden J, Buis B. Continuous measurement of left ventricular volume in animals and humans by conductance catheter. Circulation. 1984;70:812-23.

26. Ito H, Takaki M, Yamaguchi H, Tachibana H, Suga H. Left ventricular volumetric conductance catheter for rats. Am J Physiol Heart Circ Physiol. 1996;270:H1509-14

27. Suga H, Hayashi T, Suehiro S, Hisano R, Shirahata M, Ninomiya I. Equal oxygen consumption rates of isovolumic and ejecting contractions with equal systolic pressure-volume areas in canine left ventricle. Circ Res. 1981;49:1082-91.

28. Marx SO, Reiken S, Hisamatsu Y, Jayaraman T, Burkhoff D, Rosemblit N, Marks AR. PKA phosphorylation dissociates FKBP12.6 from the calcium release channel (ryanodine receptor): Defective regulation in failing hearts. Cell. 2000;101:365-76

29. Nakajima-Takenaka C, Shimizu J, Tohne K, Sakata S, Takaki M. Left ventricular lusitropic dysfunction of isoproterenol-induced hypertrophied rat heart. J Physiol Sci. 2007;57 (Suppl):S209

30. Chen-lzu Y, Chen L, Bányász T, McCulle SL, Norton B, Scharf SM, Agarwal A, Patwardhan A, Izu LT, Balk CW. Hypertension-induced remodeling of cardiac excitation-contraction coupling in ventricular myocytes occurs prior to hypertrophy development. Am J Physiol Heart Circ Physiol. 2007;293:H3301-10 